\title{
Food safety assessment on knowledge, attitude, and practice: A study among food handlers
}

\author{
Muhammad Ikmal Bin Norshaidi' ${ }^{1}$, Muhammad Arif Aizat Bashir ${ }^{2}$, Ahmad Fauzan \\ Badiuzaman $^{3}$, Sperico Michael Alden ${ }^{4}$, Mohd Yazid Ani ${ }^{5}$ \\ \{ikmalplc@gmail.com ${ }^{1}$, arif.aizat@uitm.edu.my ${ }^{2}$, fauzanb@uitm.edu.my ${ }^{3}$, \\ sperico.michael@uitm.edu.my ${ }^{4}$,mohdyazidani@gmail.com ${ }^{5}$ \} \\ 1,2,3,4,5 Faculty of Hotel and Tourism Management, Universiti Teknologi MARA Cawangan Pulau Pinang, \\ 13500 Permatang Pauh, Pulau Pinang, Malaysia
}

\begin{abstract}
Good personal hygiene and food safety practices are crucial in foodservice establishments. It should guarantee the food is safe to be eaten by the consumers. However, food poisoning cases are still arising from time to time. Therefore, this study was conducted to assess the knowledge, attitude, and practices of food safety among food-handlers. A quantitative method is applied in this study involving 140 foodhandlers. The results showed that respondents do possess the right attitude; however, there are some vital aspects of food safety that still can be improvised. In addition, the respondents were reported to have good knowledge of food handling. In terms of practice, the respondents do practice the important aspects of food safety and hygiene, but still, there are some of them that are being neglected. Hence, the outcomes of this study may perhaps increase the awareness of food-handlers regarding the importance of hygiene in food premises.
\end{abstract}

Keywords: KAP, food safety, food-handler, hipster café, quantitative research

\section{Introduction}

Malaysia is one of the most popular countries that are well known in term of food. Malaysia has a variety of races that come from different culture and background such as Malay, Chinese, Indian and ethnic Borneo. This indirectly creates a variety of culinary styles and foodways that attract people from inside and outside of the country to try.

In the foodservice area, many factors are identified to be the cause of foodborne illness such as the unknown source of material, low level of cooking skill, improper equipment used and poor personal hygiene [1]. Moreover, inappropriate food preparation, unclean restaurant, low personal hygiene, and wrong practices during foodservice operation has also been reported to cause foodborne illness [2].

Along with the above notion, therefore, this study is conducted empirically to assess the level of knowledge, attitude, and practices of food safety among the food-handlers with the aims to reveal the roots of continuing problems on food safety for possible future improvements. 


\section{Literature Review}

\subsection{Contamination in food}

Food is a substance that is high in nutrients and it is essential by microorganisms in order for them to grow and reproduce. However, due to the error or carelessness during the handling and production of food, contamination happens. It can be transmitted through air, water, unclean equipment, pest, and from the food-handlers itself. As a result, contamination that will cause foodborne illness could occur among the consumer.

Therefore, it is crucial to consider the fundamental of food safety knowledge and practices among food-handlers to minimize a foodborne outbreak. In order to bring the changes, positive attitude plus education on food safety and hygiene should be given, and it should be compulsory to all food-handlers in food production businesses. Hence, foodhandlers should equip themselves with ample knowledge and skill in order to prevent foodcaused disease.

\subsection{Food-Handlers' Knowledge about Food Safety}

Food service in food premises should guarantee the public's food safety since it is the final stage in food preparation and operational food chain. Unfortunately, many studies have shown that the greatest numbers of foodborne diseases are caused by the foodservice industry itself [3][4].

Reference [5] pointed out that although training does not always lead to a positive change in the behavior of food handling, training and education are essential to ensure that workers have the necessary awareness and knowledge to comply with food hygiene requirements.

\subsection{Food-Handlers' Attitude toward Food Safety}

In addition to knowledge, attitude is also an important factor in reducing the tendency in foodborne illness. Reference [6] stated that positive behavior, attitudes and continued education in the sustaining of safe and hygienic food-handling practices among food-handlers are correlated. Safe and hygienic food handling is an important part of the responsibilities of food-handlers and it is important for them to learn more about food hygiene. Food-handlers should wear caps, masks, and gloves to reduce the risk of contamination of food products.

\subsection{Food-Handlers' Hygiene Practices}

According to reference [7], food-handlers should tie long hair and wear appropriate hair coverings (hairnets or toques), shorten their nails and avoid nail polishing. They should remove all jewelry and makeup during food-handling. In addition, reference [8] found that there is a higher value in the present work, in which 81.5 percent of the handlers had long nails and/or had nail polish and jewelry, while 51.9 percent reported performing routine activities with respiratory or skin infections, which is in violation of current sanitary legislation norms. In addition, reference [7] determines that people who are known or suspected of having any disease that may be transmitted by food are prohibited from handling any food items or from participating in food production processes. 


\subsection{Hipster Café in Ipoh}

The Malaysian hipster cafés are normally recognized by the creative name used in food and beverages, the ingredients used and their unique presentation. While there is no agreement on its definition, the food of hipsters refers to the type of food that is produced by incorporating the latest food trends, fashions and creating its identity outside the cultural mainstream.

\section{Methodology}

\subsection{Research design}

As the objective of this study is to assess the knowledge, attitudes, and practices concerning food safety among food-handlers who are regularly involved in food service in restaurants, a descriptive research design using a quantitative approach through crosssectional studies was considered to be the suitable study method. This study also carried out cross-sectional measurements in which all the variables of the study were measured at the same time.

\subsection{Population and Sample Size}

With reference to the Ipoh City Hall Licensing Department data, there are approximately 217 foodservice premises listed as a café in Ipoh, Perak. A minimum of 140 samples will represent a population of 217 food services facilities statistically. The total number of foodhandlers was determined to be 140 samples by taking one representative from each food facility as the respondent. The researcher, therefore, obtained 140 samples in this study and this amount is considered sufficient, reliable and meaningful for rigorous analysis and results.

\subsection{Research Instrument}

The researcher uses five-point Likert scale for each question in each section of the questionnaire, allowing respondents to express themselves better and at the same time to increase the response rate and the quality of the data obtained [9].

For the content of the instrument, four (4) sections were created (section A, B, C, and D) with section (A) focusing at the first variables which are the Attitude of food-handlers. Section (B) touches on the Knowledge of food-handlers while section (C), on the other hand, assesses the practices among food-handlers. Finally, section (D) is looking at the respondents' socio-demographic profile.

\subsection{Data collection process}

Survey respondents consist of food-handlers at the local restaurant in Ipoh, Perak. The main areas of Ipoh are chosen based on the availability of restaurants in the area around Ipoh. The data collection process took about one week (1 November to 8 November 2018). The data collection process was administered personally by the researcher. The raw data was then coded according to the items and analyzed using the Social Science Statistical Package (SPSS) version 20. 


\subsection{Data analysis procedure}

Descriptive statistics were used in this study to determine the frequencies of the profile of the respondents. The mean score and standard deviation were then determined from each item in the questionnaire. Mean score and standard deviation were used in the descriptive analysis to describe the characteristics of the sample population studied and the level of knowledge, attitude and practice of food safety among food-handlers.

\section{Findings}

\subsection{Reliability coefficient}

Prior to the analysis addressing the study's objectives and research questions, the reliability test was carried out. The dimension of attitude for the food-handler based on the coefficient alpha is in good range with a coefficient value of .572. For the food-handler knowledge, the scale displays the coefficient value of .702. While for the section (C), the coefficient alpha ranges with a coefficient value of .586 .

\subsection{Respondent profile}

It is important to examine the overall dimensions of the respondents' profile before further statistical procedures. The first frequency test examines the gender of the respondents. More than half of the respondents were male with 70.71 percent $(\mathrm{n}=99)$ compared with 29.29 percent $(n=41)$ of the respondent's total number. These results showed that more males are working as food-handlers as compared to females.

In terms of age, most food-handlers were between 18 and 20 years which is 45 percent $(n=63)$, followed by 40 percent $(n=56)$ food-handlers aged between 21 and 25 . In addition, 5.71 percent $(\mathrm{n}=8)$ were 26 to 30 years old, $7.86 \%(\mathrm{n}=11)$ of food-handlers were $30-35$ years old and only $1.43 \%(\mathrm{n}=2)$ of food-handlers were between 36 and 40 years old.

For education background, food-handlers who responded in this study were found to have a lower level of education background (STPM and lower) presenting 30 percent $(n=41)$, while 70 percent $(n=99)$ of them were with higher education level (diploma and above). It is reported that more than half of the respondent were inexperienced, which amounted to 63.57 percent $(n=89)$ compared to 36.43 percent $(n=51)$ have experience in food handling.

\subsection{Analysis of respondent attitude}

As such, most of the respondents agreed that working area should be clean and safe before they start working $(M=4.70, S D=.584)$, and most of the respondents also agreed that hand washing is important before start working $(M=4.69, S D=.574)$. This indicates that most of the time respondents understand the importance of handwashing.

This is in line with the third statement where respondents clarify that unclean hand should be avoided $(M=4.66, S D=.664)$. Most of the respondents agreed with the statement of smoking while working should be avoided $(M=4.36, S D=.983)$, and hair, face, nose, etc. should not be rubbed while working $(M=4.05, S D=.962)$.

Most of the respondents disagreed that the same towel can be used to clean many places in the kitchen $(M=2.44, S D=1.293)$. Respondents slightly disagreed using a separate utensil when preparing raw and cooked food $(M=2.57, S D=1.194)$. The result also indicated food that already being defrosted should not be refrozen has the mean score of $(M=2.03)$ with a 
standard deviation of $(S D=1.073)$. Next, most of the respondent agreed that food should not be touched with a wounded hand $(M=4.06, S D=.786)$.

Overall, the result of respondent's attitude regarding food safety indicate that the respondents perform the right attitude $(M=3.232, S D=.40646)$; however, there are some vital aspects in food safety can be improvised by food-handlers.

\subsection{Analysis of respondents' knowledge}

This section portrays the mean score of the knowledge dimension, which also discourses the second objective and research question of the study. Viewing at pattern shown in Table 2, most of the respondents agreed with all the statement given. As such, food that is prepared in advance is most probably will contribute to food poisoning $(M=3.48)$ with a standard deviation $(S D=1042)$.

Wearing proper gloves while handling food reduces the risk of cross-contamination had a mean score of $(M=4.41)$ with a standard deviation $(S D=.767)$. Proper use of cap or hairnet, masks, protective gloves, and adequate clothing can minimize the risk of food contamination mean score was $(M=4.44)$ with a standard deviation of $(S D=.798)$. Most of the respondents agreed on the importance to know the correct temperature of the refrigerator or freezer to reduce the risk of food spoilage $(M=4.15, S D=.786)$. Lastly, inappropriate storage of foods (raw, cooked and ready-to-eat) may cause health hazard to consumers mean score was $(M=4.37)$ with a standard deviation of $(S D=.743)$. The entire mean score pattern indicates that respondents have a decent knowledge regarding food safety and hygiene.

Based on the observation toward the result, the respondents do possess good knowledge of food handling with an overall mean score was $(M=3.89)$ with a standard deviation $(S D=.45573)$.

\subsection{Analysis of respondents' Practice}

The final descriptive analysis is looking at respondents' practices regarding food safety. Most of the respondents agreed that they should clean and organize the workstation before they start working $(M=4.30, S D=.727)$. In addition, most of them aware that they should wash their hand first before start working and after using the washroom $(M=4.57, S D=.680)$. Using a tissue after coughing and sneezing and after that hand should be washed had a mean score of $(M=4.00)$ with a standard deviation $(S D=.929)$.

Next, the mean score for not separating cooked food and raw food during preparation was $(M=2.04)$ with a standard deviation of $(S D=1.168)$. Using the separate utensils to prepare cooked food and raw food mean score was $(M=3.55)$ with a standard deviation of $(S D=1.337)$. Never refreeze defrosted food has the mean score $(M=3.18)$ with a standard deviation $(S D=1.140)$.

Overall, the result on respondent's practice regarding food safety $(M=3.60, S D=.54276)$ indicate that the respondents do practice the important aspects in food safety and hygiene, but still, there are some of them that are being neglected by food-handlers.

\section{Conclusion}

The study findings clearly stated that the respondents did portray the good performance of attitudes by cleaning the work area, washing hands, avoiding work with unclean hand, avoiding smoking, avoiding rubbing or touching hair, face, and nose while working, avoiding handling food with a wounded hand, and avoiding wear of jewelry when handling food. This 
is because, in order to ensure the quality and the cleanliness of the food, good attitudes must be employed. Although most of the result stated the positive side, there are still certain aspects that must be looked at. Moreover, based on the results of mean scores on the respondent's knowledge regarding food safety, they showed an overall good level of knowledge regarding the matter. This indicates that food-handlers are aware of the importance of food safety. With the adequate knowledge, they basically had an idea of personal hygiene and proper food handling procedures. This will somehow encourage them to embedded good attitude and practice of food safety.

In conclusion, apart from the importance of the findings, it is imperative to emphasize several aspects of food safety and hygiene. Food safety is a critical necessity and is of primary importance in all food facilities. Food-handlers should, therefore, be trained and well-informed regarding food safety and hygiene. Restaurant owners or managers should also help in the control and monitoring of their employees.

Acknowledgments. Special thanks to Universiti Teknologi MARA Cawangan Pulau Pinang, particularly in person; Muhammad Ikmal Bin Norshaidi Muhammad 'Arif Aizat Bashir, Ahmad Fauzan Badiuzaman, Sperico Michael Alden and Mohd Yazid Ani for exceptional efforts as well as guidance in producing this paper.

\section{References}

[1] M. B. Egan et al., "A review of food safety and food hygiene training studies in the commercial sector," Food Control, 2007.

[2] N. A. Abdul-Mutalib, M. F. Abdul-Rashid, S. Mustafa, S. Amin-Nordin, R. A. Hamat, and M. Osman, "Knowledge, attitude and practices regarding food hygiene and sanitation of food handlers in Kuala Pilah, Malaysia," Food Control, 2012.

[3] S. B. Cavalli and E. Salay, "People management in foodservice establishments and food safety," Rev. Nutr., 2007.

[4] D. A. Clayton and C. J. Griffith, "Observation of food safety practices in catering using notational analysis," Br. Food J., 2004.

[5] P. Seaman and A. Eves, "Food hygiene training in small to medium-sized care settings," Int. J. Environ. Health Res., 2008.

[6] M. Howes, S. McEwen, M. Griffiths, and L. Harris, "Food handler certification by home study: Measuring changes in knowledge and behavior," Dairy, food Environ. Sanit. a Publ. Int. Assoc. Milk, Food Environ. Sanit., 1996.

[7] CAC - Codex Alimentarius Comission, "General Principles of Food Hygiene. CAC/RCP 1-1969 Rev. 4,” Codex Alimentarius Comission. 2003.

[8] A. K. C. Campos, Â. M. S. Cardonha, L. B. G. Pinheiro, N. R. Ferreira, P. R. M. de Azevedo, and T. L. M. Stamford, "Assessment of personal hygiene and practices of food handlers in municipal public schools of Natal, Brazil," Food Control, 2009.

[9] E. Babakus and W. G. Mangold, "Adapting the SERVQUAL scale to hospital services: an empirical investigation.," Health Serv. Res., 1992. 